Check for updates

Cite this: RSC Adv., 2017, 7, 45978

\title{
Novel amphiphilic glucose-responsive modified starch micelles for insulin delivery $\dagger$
}

\author{
Na Wen, (D) Chunmei Gao, Shaoyu Lü, (D) * Xiubin Xu, Xiao Bai, (D) Can Wu, Piao Ning, \\ Shaofei Zhang and Mingzhu Liu*
}

The high $\mathrm{p} K_{\mathrm{a}}$ (8.26 to 8.6 ) of PBA has restricted its glucose-responsiveness in physiological conditions, and the high cytotoxicity of polymers is also a limiting problem in their potential application for insulin delivery. Novel amphiphilic glucose-sensitive dialdehyde starch polymers containing 3-aminophenylboronic acid (APBA) as a glucose-responsive group and mPEGylated dialdehyde starch (mPEG-DAS) with hydrophobic 7-hydroxycoumarin-4-acetic acid (Cou) were synthesized. This dialdehyde starch derivative can selfassemble into mPEG-DAS-APBA-Cou micelles with "shell-core" structures in phosphate-buffered saline solution (PBS). In addition, the drug-loaded micelles can release insulin rapidly in response to hyperglycemia in a physiological environment. The results demonstrated that the mPEG-DAS-APBACou micelles showed notable glucose responsive behavior near the physiological range. The insulin release from the nanocarriers is sensitive to different concentrations of glucose, releasing insulin rapidly under the conditions of $3 \mathrm{mg} \mathrm{mL}^{-1}$ glucose while demonstrating comparatively inert release at $1 \mathrm{mg} \mathrm{mL}^{-1}$ glucose ( $\mathrm{pH}$ 7.4). MTT assays and hemolysis studies both confirmed that the mPEG-DASAPBA-Cou micelles have low cytotoxic activity to A549 cells and low blood toxicity. These results suggest that the glucose-sensitive dialdehyde starch micelles (mPEG-DAS-APBA-Cou) have potential applications as a glucose-responsive material for insulin delivery.

Received 27th July 2017

Accepted 7th September 2017

DOI: 10.1039/c7ra08291f

rsc.li/rsc-advances carriers; however, they encounter the dilemma of possible burst release of the drug in the blood, leading to hypoglycemia. In addition, the gels are large in size, up to micron dimensions, and are easily removed by the permeability and retention effect (EPR), which is undesirable for long circulation. ${ }^{\mathbf{1 0}}$ Particularly, self-assembled glucose-responsive micelles ${ }^{7,11-13}$ and vesicles ${ }^{\mathbf{8 , 1 4}}$ with PBA-containing polymers have shown uncommon and notable properties.

Compared to gels, which lack protection from a corona layer, ${ }^{15}$ self-assembled micelles are formed with the hydrophilic groups of PEG as an outer shell that is resistant to the approach of proteins to the surfaces of the nanocarriers; this shields the nanocarriers from recognition by the body's immune system ${ }^{\mathbf{1 6 , 1 7}}$ and increases blood circulation time in vivo by the protection of PEG, providing potential applications as drug carriers for insulin delivery. ${ }^{15,18-20}$ Kim et al. ${ }^{21,22}$ synthesized a new class of polymers possessing styreneboroxole and $\mathrm{N}$-functionalized maleimide with sugar-responsive behavior. The boroxole-based monomer bound to oligo (ethylene glycol) groups as a chaintransfer agent adjusted to changes in glucose solubility and demonstrated sensitive behavior at reduced glucose concentrations close to physiological conditions. Shi et al. ${ }^{7}$ developed drug-loading micelles, which were self-assembled by covalent complexation of phenylboronic acid and glycosyl. The sensitive behavior of the complex micelles required the protection of PEG. Shi et al. ${ }^{23}$ also reported micelles with favourable
State Key Laboratory of Applied Organic Chemistry, Key Laboratory of Nonferrous Metal Chemistry and Resources Utilization of Gansu Province, Department of Chemistry, Lanzhou University, Lanzhou, 730000, People's Republic of China. E-mail: lshy@lzu.edu.cn; mzliu@lzu.edu.cn

$\dagger$ Electronic supplementary information (ESI) available: Characterization of dialdehyde starch, FTIR spectra of starch and dialdehyde starch derivatives and glucose-responsiveness of different ODs of mPEG-DAS-APBA micelles in the presence of glucose with $2 \mathrm{~g} \mathrm{~L}^{-1}$ Glu. See DOI: $10.1039 / \mathrm{c} 7 \mathrm{ra08291f}$ 
responsiveness based on polyethylene glycol-co-poly(acrylic acid) with modified APBA (PEG-PAA-PBA); the lack of degradation behavior of PAA and PBA limited their potential for degradation behavior in vivo. It is necessary to adopt biodegradable and biocompatible materials, such as natural polysaccharides, in place of refractory fragments.

The medical treatment for diabetes is a persistent process; hence, it requires baseline drugs that are non-toxic, show sustained release, are biodegradable, and are non-toxic to the body for in vivo applications. However, the compatibility between favourable glucose-responsiveness and high biodegradability and biocompatibility is a limitation for drug delivery in current studies. Therefore, designing glucose-sensitive materials is imminently and greatly needed to achieve high biodegradability and biocompatibility in insulin delivery systems. ${ }^{24}$ Starch, a major natural source of polysaccharides, exhibits nontoxicity, compatibility, and nonimmunogenicity. Thus, it has been widely used in the pharmaceutical field as an excipient for drug delivery systems. ${ }^{25,26}$ Starch has superior advantages of low cost and biodegradability due to hydrolysis and human enzymes in comparison to other polysaccharides. ${ }^{27}$ In addition, various functional materials can be attained via modification of starch due to the large numbers of functional hydroxyl groups in the chains. ${ }^{28,29}$ The use of dialdehydes has been explored to obtain more biocompatible materials; ${ }^{30}$ aldehyde-functionalized starch has aroused considerable interest due to its minimal toxicity. ${ }^{31}$ The preparation of dialdehyde starches containing aldehyde groups has been achieved by reaction with sodium periodate $\left(\mathrm{NaIO}_{4}\right)^{32,33}$ It is noteworthy that micelles can be prepared via self-assembly of amphiphilic starch derivatives. ${ }^{29,34,35}$ Lehr et al. ${ }^{27}$ prepared starch-graft-polyethylenimine copolymers for efficient biodegradable gene delivery. Zhang et $a .^{36}$ synthesized grafted copolymers by a reaction between starch and poly(L-glutamic acid) with $\mathrm{pH}$-responsive behavior for controlled release of insulin; this is a fascinating option for synthetic polymers in drug delivery systems.

For most reports focusing on glucose-responsiveness, the high $\mathrm{p} K_{\mathrm{a}}$ (8.26 to 8.6) of PBA has restricted its practical applications. ${ }^{37,38}$ However, researchers have attempted to decrease the $\mathrm{p} K_{\mathrm{a}}$ of PBA. For example, Yoon et al. ${ }^{39}$ reported a PBAcontaining complex with polyol polymer, a stronger acid, and decreased the $\mathrm{p} K_{\mathrm{a}}$ of the PBA derivative. Wu et al..$^{40}$ introduced amino groups into polymers to enhance the binding affinity of nitrogen and boron and to self-regulate insulin release, even responding positively to glucose in physiological conditions. A subtle relationship may exist in polymers between the structure of coumarins and the $\mathrm{p} K_{\mathrm{a}}$ of PBA. Therefore, PBA-based materials are susceptible to glucose-responsiveness at lower $\mathrm{pH}$ values than the $\mathrm{p} K_{\mathrm{a}}$ of PBA.

Herein, a series of dialdehyde starch derivative polymers (mPEG-DAS-APBA-Cou) were prepared by grafting PEG as the hydrophilic outer shell, Cou as the hydrophobic core and PBA as the glucose-responsive group to a dialdehyde starch backbone. Introducing Cou may decrease the $\mathrm{p} K_{\mathrm{a}}$ of a material, and the starch backbone mainly enhances its biodegradable behavior. The polymers were able to self-assemble into spherical micelles in PBS solution. The polymers showed rapid response to

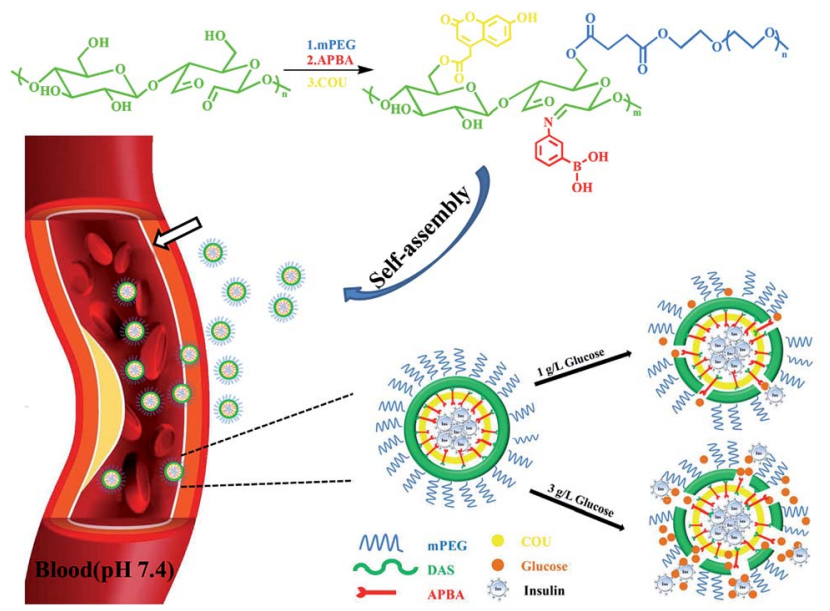

Scheme 1 The formation of insulin-loaded core-shell mPEG-DASAPBA-Cou micelles and their insulin release behavior under different concentrations of glucose in the blood microenvironment.

glucose due to the hydrophobic micelle core and the apparently low $\mathrm{p} K_{\mathrm{a}}$ of $\mathrm{PBA}$ in physiological conditions, as illustrated in Scheme 1. The micelles quickly disassembled to release insulin because of the sensitivity of the PBA-based polymer to different concentrations of glucose in the blood microenvironment. The safety and biocompatibility of the micelles as drug carriers were also confirmed by cytotoxicity and hemolysis tests.

\section{Experimental}

\section{Materials}

Soluble starch $\left(M_{\mathrm{w}} 8.8 \mathrm{kDa}\right)$ was provided by the Zhejiang Linghu Chemical Reagent Factory. Polyethylene glycol monomethyl ether (mPEG, $\quad M_{\mathrm{n}} \quad 1.9 \quad \mathrm{kDa}$ ), 1-ethyl-3-(3-dimethylaminopropyl) carbodiimide hydrochloride (EDC $\cdot \mathrm{HCl})$, 7-hydroxycoumarin-4acetic acid, 3-aminophenylboronic, insulin from porcine pancreas and pyrene were purchased from Aladdin Chemistry Co., Ltd. Succinic anhydride, $N$-hydroxysuccinimide, and 4dimethylaminopyridine (DMAP) were purchased from J\&K Reagent Company. All other chemicals were of analytical grade and were used without further purification.

\section{Synthesis and characterization of dialdehyde starch derivatives}

Synthesis of mPEGylated modified dialdehyde starch (mPEG-DAS) and APBA-functional dialdehyde starch copolymers (mPEG-DAS-APBA). The mPEGylated dialdehyde starch (mPEG-DAS) was conveniently synthesized by conjugating mPEG$\mathrm{COOH}$ with different oxidation degrees (ODs) of DAS according to previous reports. ${ }^{41,42}$ Briefly, DAS with different ODs (0.2 g), mPEG$\mathrm{COOH}(0.38 \mathrm{~g})$, EDC $\cdot \mathrm{HCl}(0.23 \mathrm{~g})$ and DMAP $(0.05 \mathrm{~g})$ were dissolved in DMSO and stirred for $48 \mathrm{~h}$ under ambient conditions. Then, to remove DMSO and unreacted substances, the mixture was dialyzed in a dialysis bag (MWCO $10 \mathrm{kDa}$ ) for three days. Finally, the solution was lyophilized and the target product mPEGylated dialdehyde starch (mPEG-DAS) was obtained. 
mPEG-DAS (0.2 g) and APBA (0.1 g) were dissolved in DMSO and heated to $60{ }^{\circ} \mathrm{C}$ for $6 \mathrm{~h}$ under nitrogen atmosphere. Then, the mixture was purified in a dialysis bag (MWCO $3.5 \mathrm{kDa}$ ) for three days and lyophilized to obtain the product APBAfunctional dialdehyde starch (mPEG-DAS-APBA).

Synthesis of Cou-ornamented mPEG-DAS-APBA copolymers (mPEG-DAS-APBA-Cou). Copolymers containing the carboxyl groups of Cou were conveniently coupled with the hydroxyl groups of mPEG-DAS-APBA in the presence of EDC/NHS as described previously. ${ }^{43}$ Briefly, mPEG-DAS-APBA (0.1 g) and Cou $(0.1 \mathrm{~g})$ were dissolved in DMSO containing EDC $(0.15 \mathrm{~g})$ under stirring at $45^{\circ} \mathrm{C}$ for $12 \mathrm{~h}$. After that, NHS (0.03 g) was added to the above solution. The solution was purified by dialyzing against a deionized water/methanol mixture $(1 / 4 \mathrm{v} / \mathrm{v})$ for 2 days, followed by dialyzing against deionized water for 3 days using dialysis bags (MWCO $3.5 \mathrm{kDa}$ ). Finally, Cou-ornamented mPEGDAS-APBA (mPEG-DAS-APBA-Cou) was obtained by freezedrying.

Characterization of dialdehyde starch derivatives. Dialdehyde starch derivatives were characterized by ${ }^{1} \mathrm{H}$ NMR spectra, which were recorded on a Bruker AVANCE III $400 \mathrm{MHz}$ spectrometer (Germany), and by FTIR spectra, which were recorded on an Agilent Technologies Cary 600 Series FTIR spectrometer (USA) at room temperature.

\section{Preparation and characterization of dialdehyde starch derivative micelles}

Micellisation of dialdehyde starch derivatives. The micelles of MPEG-DAS-APBA and MPEG-DAS-APBA-Cou were prepared according to literature procedures. ${ }^{44}$ Herein, $20 \mathrm{~mL}$ of PBS was slowly dropped into solutions of the mPEG-DAS-APBA and mPEG-DAS-APBA-Cou polymers (20 mg) under gentle shaking at $37^{\circ} \mathrm{C}$ over $3 \mathrm{~h}$. Then, the solution was sonicated for $10 \mathrm{~min}$ to obtain an optically transparent solution. Finally, the micelles solution was passed through $0.45 \mathrm{~nm}$ membrane filters and stored at room temperature.

Characterization of dialdehyde starch derivative micelles. The critical micelle concentration (CMC) was estimated using pyrene as a probe by fluorescence spectrophotometry; the spectra were recorded on a Perkin-Elmer LS55 (USA) instrument at room temperature. Micelle solutions with different concentrations ranging from $10^{-4}$ to $10^{-1} \mathrm{mg} \mathrm{mL}^{-1}$ were prepared. The fluorescence measurements were acquired at an emission wavelength of $395 \mathrm{~nm}$, and the excitation was monitored from 350 to $400 \mathrm{~nm}$. Dynamic light scattering (DLS) was used to measure the sizes of the micelles. The zeta potentials of the particles in different $\mathrm{pH}$ solutions were determined with a Malvern Zetasizer Nano S instrument (Malvern Instruments Ltd.). The morphologies of the micelles were examined using TEM, which was performed with a JEM-2100F instrument (JEOL, FEI, Japan).

\section{In vitro stability study of micelles}

The stabilities of the micelles were tested by dispersion in PBS solution ( $\mathrm{pH}$ 7.4) and gentle shaking at $100 \mathrm{rpm} \min ^{-1}$ at physiological temperature. The diameters of the micelles were measured at intervening times ( $4 \mathrm{~h}, 8 \mathrm{~h}, 24 \mathrm{~h}$ and $48 \mathrm{~h})$ using DLS.

\section{Glucose-responsiveness of the complex micelles}

The $\mathrm{p} K_{\mathrm{a}}$ values of the complex micelles were determined by UV-vis according to a reported method. ${ }^{45}$ The glucose-sensitivity behaviors of the mPEG-DAS-APBA and MPEG-DAS-APBA-Cou micelles in PBS were measured by DLS. The concentrations of glucose solution were 0,1 and $3 \mathrm{mg} \mathrm{mL}^{-1}$, referring to the final diluted concentration after adding glucose to the micelle solutions. The micelle solutions were prepared for DLS analysis by filtering as mentioned.

\section{Hemolysis assay}

The membrane disruption of red blood cells (RBCs) can be employed to evaluate the blood toxicity of polymers. Fresh human blood samples were obtained from Lanzhou University first affiliated hospital and were centrifuged at $1600 \mathrm{rpm} \mathrm{min}^{-1}$ for $5 \mathrm{~min}$; all experiments were performed in compliance with the approval of the Institutional Authority for Laboratory Animal Care. A549 cells were derived from an assay on rabbit mesenchymal stem cells. Afterwards, the micelle groups were prepared by adding $0.2 \mathrm{~mL}$ RBC supernatant to $0.8 \mathrm{~mL}$ micelle solution $\left(1 \mathrm{mg} \mathrm{mL}^{-1}\right)$. Then, the micelle systems were incubated at $37{ }^{\circ} \mathrm{C}$ for $2 \mathrm{~h}$. Furthermore, the micelle systems were centrifuged at $1600 \mathrm{rpm} \mathrm{min}^{-1}$ for $5 \mathrm{~min}$. Finally, the supernatant $(0.5 \mathrm{~mL})$ was added to $5 \mathrm{~mL} \mathrm{PBS}$, and the percentages of hemolysis of the RBCs with various samples were calculated via the absorbance of released hemoglobin at $570 \mathrm{~nm}$ by UV-vis spectrophotometer (Perkin-Elmer Lambda 35, USA) based on the following equation:

$$
\% \text { hemolysis }=\frac{A_{\text {sample }}-A_{\text {negative }}}{A_{\text {positive }}-A_{\text {negative }}} \times 100 \%
$$

where $A_{\text {sample }}, A_{\text {negative, }}$ and $A_{\text {positive }}$ represent the absorbances measured from the sample, PBS, and water groups, respectively.

\section{Insulin loading and glucose-responsive release}

Preparation of drug-loaded micelles. The polymers (10 mg) were added to $10 \mathrm{~mL}$ insulin solution $\left(0.25 \mathrm{mg} \mathrm{mL}^{-1}\right)$ under constant stirring at $37{ }^{\circ} \mathrm{C}$. Drug-loaded micelles were obtained by dialysis for $24 \mathrm{~h}$ using dialysis bags (MWCO $8 \mathrm{kDa}$ ). The dosage of insulin was monitored by a UV-vis spectrophotometer at a wavelength of $276 \mathrm{~nm}$. The insulin drug loading efficiency (DLE) and drug loading capacity (DLC) were calculated from the following equations:

$$
\mathrm{DLE} \%=\frac{\text { encapsulated insulin }}{\text { total insulin }} \times 100 \%
$$

$$
\text { DLC } \%=\frac{\text { encapsulated insulin }}{\text { micelle weight }} \times 100 \%
$$

In vitro glucose-responsive release of insulin-loaded micelles. The release of insulin from the micelles in vitro was 
studied by dialysis. The drug-loaded micelles in PBS with different glucose concentrations of 0,1 and $3 \mathrm{mg} \mathrm{mL}^{-1}$ were incubated and gently shaken at $37{ }^{\circ} \mathrm{C}$. The released insulin was sampled using an UV-vis absorption spectrometer at $276 \mathrm{~nm}$. Cumulative release is expressed as the total percentage of drug released through the dialysis membrane over time.

\section{Circular dichroism spectroscopy}

The structure of the released insulin was determined by circular dichroism (CD) spectra, which were acquired using a DSM1000 CD spectropolarimeter (USA). A standard insulin solution of $1 \mathrm{mg} \mathrm{mL}{ }^{-1}$ was prepared in PBS (pH 7.4) for CD measurements.

\section{Cytotoxicity test}

The cell suspension in culture medium was plated at $5 \times 10^{4}$ cells per well in a 96-well plate and incubated in a humidified atmosphere with $5 \% \mathrm{CO}_{2}$ for $24 \mathrm{~h}$ at $37{ }^{\circ} \mathrm{C}$. Then, a methyl thiazolyl tetrazoliumviability assay (MTT) was carried out with A549 cancer cells from rabbit mesenchymal stem cells. The culture medium was replaced with $200 \mu \mathrm{L}$ of the prepared culture medium containing blank micelles with concentrations of $1,5,10,50$, and $100 \mu \mathrm{g} \mathrm{mL}{ }^{-1}$ for $24 \mathrm{~h}$ and $48 \mathrm{~h}$. MTT solution was added to each well, and the wells were incubated for another $4 \mathrm{~h}$. The optical density was measured using a microplate reader at $490 \mathrm{~nm}$. The cell viability was calculated by means of the percent ratio of the absorbance of the mixtures and micelles to the control.

\section{Results}

\section{Synthesis and characterization of dialdehyde starch derivatives}

In this work, we successfully synthetized amphiphilic dialdehyde starch derivatives containing hydrophilic mPEG, APBA functional groups and hydrophobic Cou. The dialdehyde starch derivatives of mPEG-DAS-APBA-Cou were synthesized via three steps (Scheme 2). Firstly, mPEG-COOH was introduced as a hydrophilic group to dialdehyde starch by an etherification reaction. Then, the conjugated polymer was readily synthesized by reacting the aldehyde groups of mPEG-DAS with PBA via Schiff bases $(-\mathrm{C}=\mathrm{N}-)$ due to its high stability. Finally, Cou as the hydrophobic group was attached by esterification reactions of the hydroxyl groups on the dialdehyde starch chains. Dialdehyde starch with different ODs was synthesized and determined by hydroxylamine hydrochloride titration and confirmed by FTIR spectra, as shown in Fig. S1 and Table S1. $\dagger$

Typical ${ }^{1} \mathrm{H}$ NMR spectra of the copolymers are presented in Fig. 1. Specifically, the glucose units and aldehyde groups of dialdehyde starch (4.99 to 5.51 and $9.26 \mathrm{ppm}$, respectively) are shown in Fig. 1B. ${ }^{46}$ The respective assigned peaks of both dialdehyde starch and mPEG could be found in their spectra. According to our published method, ${ }^{47}$ the degree of substitution (DS) of mPEG in MPEG-St polymer was calculated to be 0.25. The protons of the phenyl groups appeared in the range from 6.98 to 7.6 ppm in PBA in Fig. 1A (e, 7.46 ppm), while typical peaks of mPEG from the methylene $\left(-\mathrm{CH}_{2}-\mathrm{CH}_{2}-\right.$, b and $\left.\mathrm{c}\right)$ and

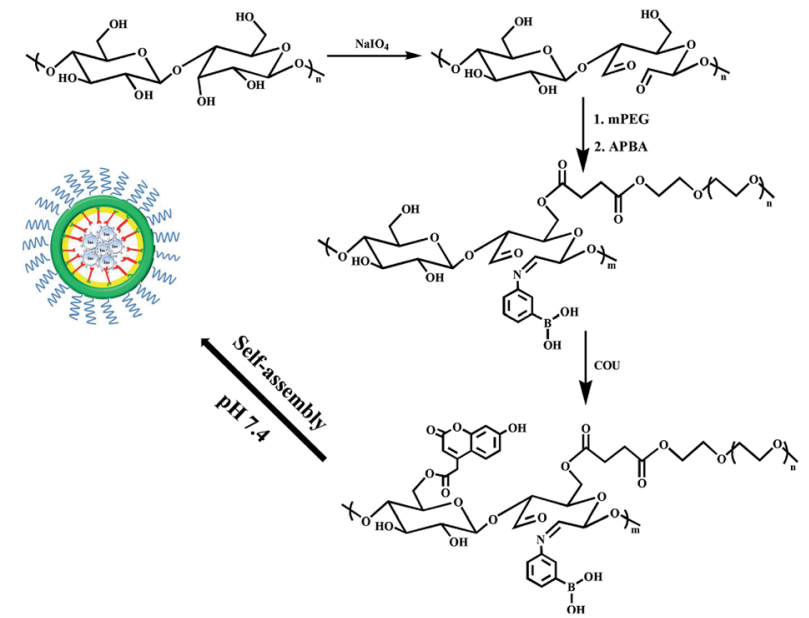

Scheme 2 The synthetic scheme of mPEG-DAS-APBA-Cou.

terminal methoxyl $\left(-\mathrm{O}-\mathrm{CH}_{3}\right.$, a) groups were observed at $3.51 \mathrm{ppm}$ and $3.34 \mathrm{ppm}$, respectively. Because Cou and PBA contain phenyl groups, the Cou-OH spectrum features absorption peaks at 3.24, as described previously. ${ }^{48}$ The DS of APBA was 0.15 according to the calculated copolymer compositions of the integrated area between the saccharide $(5.11 \mathrm{ppm})$ and APBA (7.46 ppm) signals. Similarly, the DS of the Cou signals at $3.24 \mathrm{ppm}$ was 0.1 . In order to prepare mPEG-DAS-APBA polymers with different degrees of cross-linking, a series of experiments was designed with different ODs of mPEG-DAS and APBA. The degrees of cross-linking in the mPEG-DAS-APBA polymers are listed in Table 1 . For different ODs of $20 \%, 40 \%$ and $60 \%$ of mPEG-DAS, the corresponding degrees of crosslinking were $0.08,0.15$, and 0.17 , respectively.

The FTIR spectra of St, DAS and mPEG-DAS-APBA-Cou are shown in Fig. 2. Fig. 2a shows the characteristic peaks of the St sample; a faint adsorption band can be found at $1660 \mathrm{~cm}^{-1}$ that corresponds to starch bone stretching vibrations. Compared with starch, the FTIR spectrum of Fig. 2c shows $-\mathrm{C}=\mathrm{O}-$ stretching vibrations and a new strong characteristic absorption band at $1106 \mathrm{~cm}^{-1}$ resulting from $\mathrm{C}-\mathrm{O}$ asymmetric stretching vibrations; moreover, the $-\mathrm{C}=\mathrm{O}-$ absorption peak was weaker than that in the spectrum of DAS (Fig. 2b).

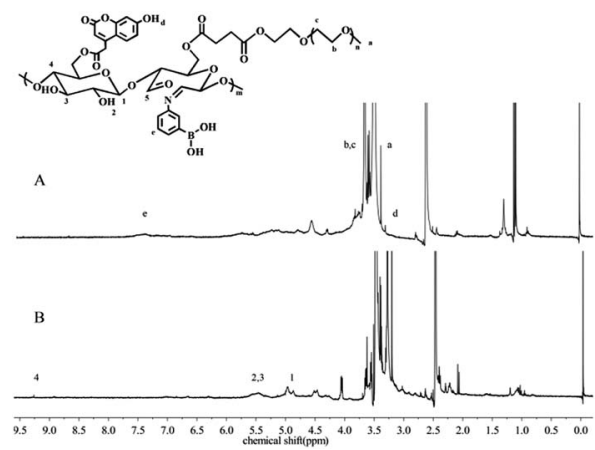

Fig. $1{ }^{1} \mathrm{H}$ NMR spectra of $40 \%$ mPEG-DAS-APBA-Cou (A) and 40\% mPEG-DAS (B) in DMSO- $d_{6}$ solution. 
Table 1 Physicochemical properties of synthesized starch derivatives

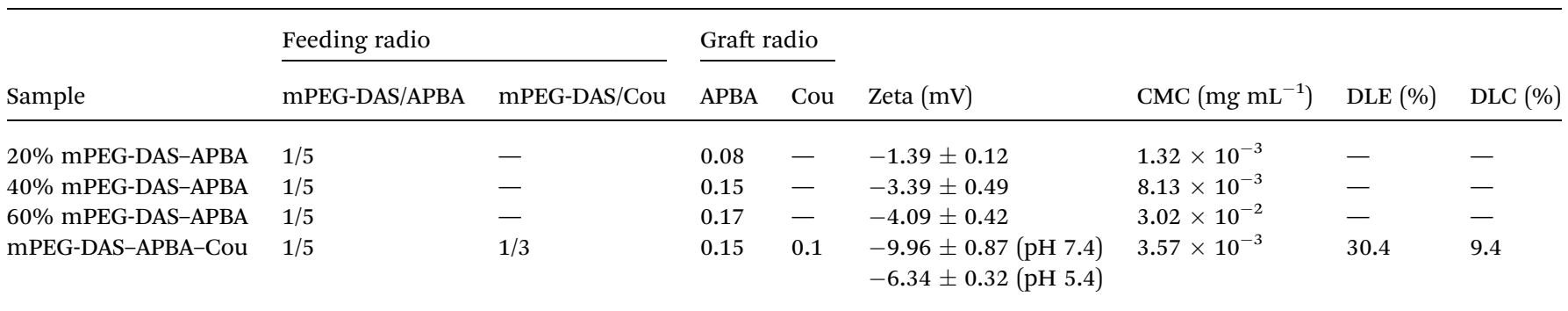

\section{Characterization of dialdehyde starch derivative micelles}

During the self-assembly process, CMC is a vital characteristic of polymers that not only indicates the self-aggregation behavior of micelles, but also evaluates their stability. ${ }^{49}$ The self-assembly behaviors of different ODs of mPEG-DAS-APBA micelles and mPEG-DAS-APBA-Cou micelles were investigated by fluorimetry. The changes in the intensity ratio of $I_{384} / I_{373}$, which are dependent upon high sensitivity to hydrophobicity, are plotted in Table 1 and Fig. 3A. It can be observed that the CMC increased with increasing value of OD.

As shown in Fig. 3, TEM and DLS were performed to evaluate the aggregation behaviors of 40\% mPEG-DAS-APBA and mPEGDAS-APBA-Cou micelles as well as their sizes and morphologies. The morphologies of the 40\% mPEG-DAS-APBA and mPEG-DAS-APBA-Cou polymeric self-assemblies were spherical, with diameters of about $175 \pm 7.5 \mathrm{~nm}$ and $113.5 \pm 3.5 \mathrm{~nm}$, respectively. On the other hand, the "core-shell" structures of the micelles with different compositions were also very distinct in the TEM observations (Fig. 3C and D). Moreover, the DLS results indicated that the hydrodynamic diameters of the $40 \%$ mPEG-DAS-APBA (241.4 $\mathrm{nm})$ and mPEG-DAS-APBA-Cou $(177.9 \mathrm{~nm})$ micelles decreased upon addition of hydrophobic Cou, where each of the groups exhibited a monomodal size distribution (Fig. 3B). The zeta potential values of the $40 \%$ mPEG-DAS-APBA and mPEG-DAS-APBA-Cou polymers with "core-shell" structures are shown in Table 1 . The zeta value of $40 \%$ mPEG-DAS-APBA is $-25.39 \pm 0.49 \mathrm{mV}$ (PBS 7.4), whereas slight changes in the zeta potential of the mPEG-DAS-APBA-

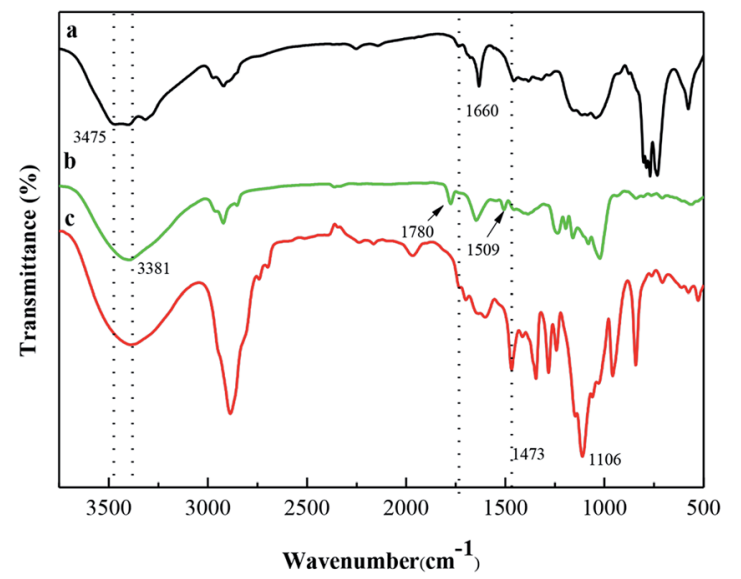

Fig. 2 FTIR spectra of St (a), DAS (b), and mPEG-DAS-APBA-Cou (c).
Cou micelles were observed at $-9.96 \pm 0.87 \mathrm{mV}$ (PBS 7.4) and $-6.34 \pm 0.32 \mathrm{mV}$ (PBS 5.4).

\section{In vitro stability study of dialdehyde starch derivative micelles}

First, the stabilities of the dialdehyde starch derivative micelles were measured by DLS. As shown in Fig. 4A, the stabilities of the 40\% mPEG-DAS-APBA and mPEG-DAS-APBA-Cou micelles were investigated at $1 \mathrm{mg} \mathrm{mL}^{-1}$ for $4 \mathrm{~h}, 8 \mathrm{~h}, 24 \mathrm{~h}$ and $48 \mathrm{~h}$ at $37{ }^{\circ} \mathrm{C}$. The $40 \%$ mPEG-DAS-APBA and mPEG-DAS-APBA-Cou micelles changed distinctly from $241.4 \mathrm{~nm}$ to around $438.2 \mathrm{~nm}$ and from $177.9 \mathrm{~nm}$ to around $307 \mathrm{~nm}$ after $48 \mathrm{~h}$, respectively. The $40 \%$ mPEG-DAS-APBA and mPEG-DAS-APBA-Cou micelles both showed slight increases in particle size after $8 \mathrm{~h}$ and considerable increases after $24 \mathrm{~h}$.

\section{Glucose-responsiveness of dialdehyde starch derivative micelles}

The UV absorbance ( $268 \mathrm{~nm}$ ) is shown in Fig. $4 \mathrm{~B}$; the $\mathrm{p} K_{\mathrm{a}}$ values of $40 \%$ mPEG-DAS-APBA and mPEG-DAS-APBA-Cou were estimated to be 8.0 and 7.6. It can be clearly seen in Fig. $4 \mathrm{C}$ that the particle size of the mPEG-DAS-APBA-Cou micelles remained almost unchanged in $70 \mathrm{~min}$ in the presence of $0 \mathrm{~g} \mathrm{~L} \mathrm{~L}^{-1}$ glucose. Notably, glucose-responsive behavior was observed at 1, 2 and $5 \mathrm{~g} \mathrm{~L}^{-1}$ in different concentrations of

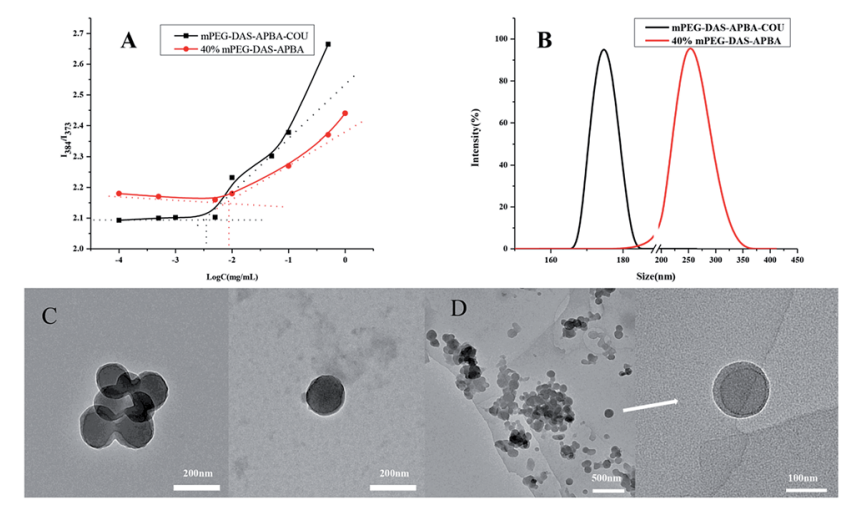

Fig. 3 Characterization of 40\% mPEG-DAS-APBA micelles and mPEG-DAS-APBA-Cou micelles. Variations of intensity ratio $\left(/ 384 / /_{373}\right)$ versus logarithm of $40 \%$ mPEG-DAS-APBA and mPEG-DAS-APBACou micelles (A), size distributions of $40 \% \mathrm{mPEG}-\mathrm{DAS}-\mathrm{APBA}$ and mPEG-DAS-APBA-Cou micelles (B), TEM image of the $40 \%$ mPEGDAS-APBA micelles (C), TEM image of the mPEG-DAS-APBA-Cou micelles (D). 

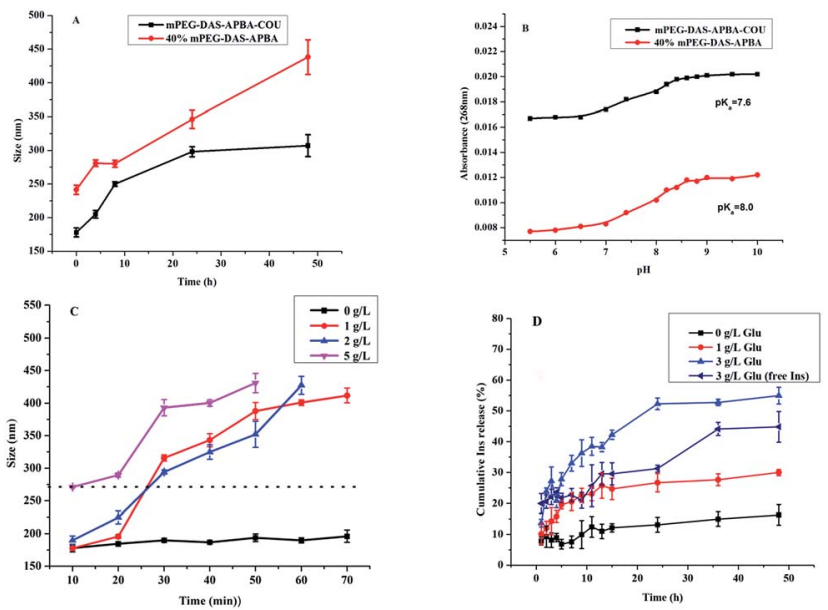

Fig. 4 In vitro stability studies of $40 \% \mathrm{mPEG}$-DAS-APBA micelles and mPEG-DAS-APBA-Cou micelles (A). The $\mathrm{p} K_{\mathrm{a}}$ values of $40 \% \mathrm{mPEG}$ DAS-APBA and mPEG-DAS-APBA-Cou were determined to be 7.6 and 8.0 by the absorbance change at $268 \mathrm{~nm}$ that occurs upon conversion from the trigonal form (low $\mathrm{pH}$ ) to the tetrahedral form (high pH) (B). Glucose-responsiveness of mPEG-DAS-APBA-Cou micelles in the presence of glucose with different concentrations of 0 , 1,2 and $5 \mathrm{~g} \mathrm{~L}^{-1} \mathrm{Glu}(\mathrm{C})$. In vitro cumulative release of insulin ( $\left.\mathrm{pH} 7.4\right)$. mPEG-DAS-APBA-Cou micelles and free insulin in different glucose media $\left(0,1\right.$ and $\left.3 \mathrm{mg} \mathrm{mL}^{-1}\right)(D)$.

glucose solution; the micelles remained stable in size in the presence of $1 \mathrm{~g} \mathrm{~L}^{-1}$ glucose and changed slightly in the presence of $2 \mathrm{~g} \mathrm{~L}^{-1}$ glucose in $10 \mathrm{~min}$. Then, the micelles completely disintegrated in 20 to $30 \mathrm{~min}$ in the presence of 1 and $2 \mathrm{~g} \mathrm{~L}^{-1}$ glucose. Finally, at a concentration of $5 \mathrm{~g} \mathrm{~L}^{-1}$ glucose, the complex micelles completely disintegrated at the outset.

\section{Hemolysis assay}

As shown in Fig. 5, compared with the positive control, the dialdehyde starch derivative micelles had lower hemolytic activity. We found that $60 \%$ mPEG-DAS-APBA showed a higher hemolytic activity value of $7.93 \%$, whereas $40 \%$ mPEG-DASAPBA $(2.00 \%)$ exhibited the lowest hemolysis value. Compared to mPEG-DAS-APBA-Cou (4.15\%), the hemolytic activity was slightly greater than that of $40 \%$ mPEG-DAS-APBA. In addition, a photograph showed that RBCs were released into the supernatant after treatment with dialdehyde starch derivative micelles. However, a slight red color was observed for the dialdehyde starch derivative micelles-treated cells.

\section{Insulin loading and glucose-responsive release}

The results are shown in Table 1. The drug loading efficiency (DLE) and drug loading content (DLC) of the insulin-loaded micelles were found to be $30.4 \%$ and $9.4 \%$ with respect to the standard curve of insulin, respectively.

Fig. 4D shows that the cumulative release of insulin-loaded mPEG-DAS-APBA-Cou polymers and free insulin upon exposure to different glucose media $\left(0,1\right.$ and $\left.3 \mathrm{mg} \mathrm{mL}^{-1}\right)$ at $37{ }^{\circ} \mathrm{C}$ (pH 7.4) increased with increasing glucose concentration. As indicated in Fig. 6A, little conformational change was detected

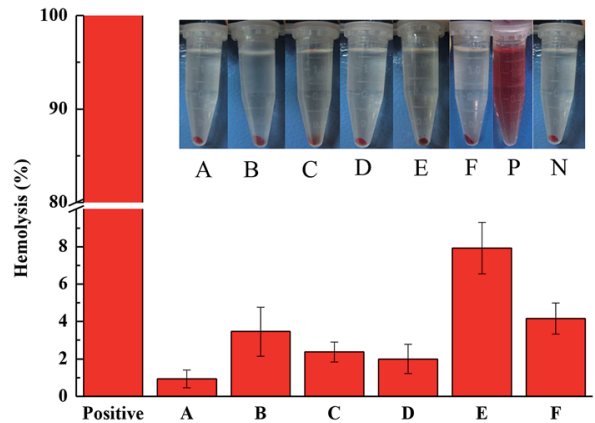

Fig. 5 Percent hemolysis of RBCs incubated with dialdehyde starch derivative micelle systems. Inset photograph: RBCs treated with 40\% DAS (A), 40\% DAS-APBA (B), 20\% mPEG-DAS-APBA (C), 40\% mPEGDAS-APBA (D), 60\% mPEG-DAS-APBA (E), and mPEG-DAS-APBACou (F) compared to water and PBS (7.4) groups. P, positive; N, negative.
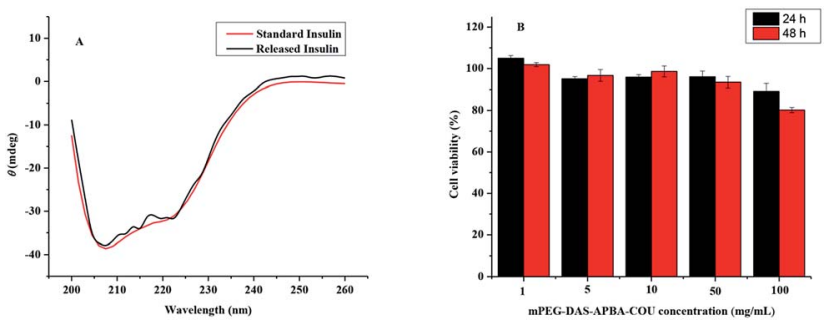

Fig. 6 UV-CD spectra of standard insulin and released insulin (A), viability of A549 lung cells after incubation with blank mPEG-DASAPBA-Cou micelles at different concentrations for $24 \mathrm{~h}$ and $48 \mathrm{~h}$ (B).

for the insulin released from mPEG-DAS-APBA-Cou compared with standard insulin ( $\mathrm{pH} 7.4)$; the $[\phi]_{208} /[\phi]_{223}$ ratios for standard insulin and released insulin were 1.24 and 1.17, respectively.

\section{Cytotoxicity test}

To evaluate the potential toxicity of the copolymers, an MTT assay was performed to measure the cell cytotoxicity of the micelles in A549 lung cancer cells for $24 \mathrm{~h}$ and $48 \mathrm{~h}$ (Fig. 6B). Fig. 6B shows that the cells were exposed to various concentrations of the blank mPEG-DAS-APBA-Cou and incubated for 24 and $48 \mathrm{~h}$. More than $80 \%$ A549 lung cancer cell viability was retained at all concentrations from 1 to $100 \mathrm{mg} \mathrm{mL}^{-1}$ after $48 \mathrm{~h}$ cultivation as the nanoparticle concentration increased.

\section{Discussion}

In the present study, we developed a novel amphiphilic glucosesensitive dialdehyde starch polymer composed of APBA and Cou with an mPEG-DAS molecular skeleton for insulin release in diabetes treatment. On the basis of the ${ }^{1} \mathrm{H}$ NMR and FTIR spectral analyses, we confirmed that the targeted dialdehyde starch derivatives were successfully synthesized. During the self-assembly process, the CMC value of 20\% mPEG-DAS-APBA provided a lower value than $60 \%$ mPEG-DAS-APBA owing to the 
different ODs based on different grafting ratios of hydrophobic PBA. The CMC value of $40 \%$ mPEG-DAS-APBA-Cou was lower than that of $40 \%$ mPEG-DAS-APBA due to the presence of hydrophobic Cou groups; a lower CMC results in higher micelle stability. ${ }^{\mathbf{5 0}}$

The morphologies of the $40 \%$ mPEG-DAS-APBA and mPEGDAS-APBA-Cou polymeric self-assemblies were spherical. The "core-shell" structures of the micelles with different compositions were also very distinct in the TEM observations. However, the micelles showed lower dispersibility in the dry state; this can be attributed to the lower ratio of hydrophilic groups grafted onto dialdehyde starch, whose chain segments tangle easily. Moreover, the DLS results indicated that the hydrodynamic diameters of the $40 \%$ mPEG-DAS-APBA and MPEG-DAS-APBACou micelles were reduced upon addition of hydrophobic Cou, where each of the groups exhibited a monomodal size distribution. The interactions of electrostatic, hydrophobic and hydrogen bonds, as well as existing intermolecular forces, may lead to micellization in aqueous solution.

The stability of the dialdehyde starch derivative micelles was measured by DLS because the physiological stability of polymeric micelles is of importance for long-term storage, transportation, and scalable processing in drug carrier applications. ${ }^{51}$ Because the micellar structure is susceptible to exposure of the hydrophobic core, the 40\% mPEG-DAS-APBA and mPEG-DAS-APBA-Cou micelles showed a slight increase in particle size after $8 \mathrm{~h}$ and $24 \mathrm{~h}$ due to the appearance of abundant aggregation of the cores. Compared with the $40 \%$ mPEGDAS-APBA micelles, the mPEG-DAS-APBA-Cou micelles indicated preferable stability. These results confirmed that the mPEG-DAS-APBA-Cou micelles have extraordinarily enhanced stability, which is propitious for a drug carrier.

As prospective self-regulated materials for insulin delivery, PBA-based polymer materials have been widely studied. However, favourable response to different glucose concentrations is difficult under physiological conditions for PBA-based nanopolymers because of the high $\mathrm{p} K_{\mathrm{a}}$ of PBA. ${ }^{37,38}$ Dialdehyde starch derivative polymers were prepared to build a platform to attach Cou, which is more acidic than the phenylboronic acid derivative in the polymer. From the UV absorbance spectra $(268 \mathrm{~nm})$ shown in Fig. $4 \mathrm{~B}$, the $\mathrm{p} K_{\mathrm{a}}$ values of $40 \%$ mPEG-DASAPBA and mPEG-DAS-APBA-Cou imply that the boronate ester would remain stable at $\mathrm{pH}$ values between 8.0 and 7.6. Particularly, the $\mathrm{p} K_{\mathrm{a}}$ value of mPEG-DAS-APBA-Cou was close to physiological conditions. As the glucose sensitivity in complexation with polyol compounds increased under physiological conditions, the $\mathrm{p} K_{\mathrm{a}}$ values of the PBA-based materials apparently decreased. ${ }^{52}$ The mPEG-DAS-APBA micelles with different ODs displayed diverse glucose-responsiveness behaviors, showing that the complex micelles with high contents of dialdehyde groups were more sensitive to glucose; this would lead to uncontrolled release, as briefly shown in Fig. S2. $\dagger$ In this study, the micelles with glucose attached indeed manifested water-solublility. These phenomena completely clarified the disintegrative process in the micelles "core-shell" structure and also indicated the high solubility of the amphipathic nanoparticles. The polymer was expected to form micelles with enhanced glucose-responsiveness for applications in selfregulated insulin delivery close to physiological range.

Determining the blood compatibility and cytotoxicity of dialdehyde starch derivative micelle systems is the primary screening for their in vivo applications. ${ }^{53}$ Because the hydrophobic polymers are combined with human blood, the possibility of absorptivity of plasma proteins will be enhanced. As a result, an embolism or thrombosis at the blood-contacting side of the polymer interface in the bloodstream introduces blood platelet activation. ${ }^{54-56}$ As shown in Fig. 5, compared with the positive control, the dialdehyde starch derivative micelles had lower hemolytic activity. Thus, these results also indicate that $40 \%$ mPEG-DAS-APBA and mPEG-DAS-APBA-Cou can prevent hemolytic activity by blocking the hydration layer around the polymer surfaces and electrostatic interactions with the RBC membrane, improving their blood compatibility. To evaluate the potential cytotoxicities of the copolymers, the cytotoxicities of the micelles toward A549 lung cancer cells were determined by MTT assays for $24 \mathrm{~h}$ and $48 \mathrm{~h}$, respectively (Fig. 6B). The blank mPEG-DAS-APBA-Cou micelles demonstrated low toxicity and good cytocompatibility with the backbone of DAS. Compared with the PBA-based material reported by Yang et al., ${ }^{11}$ blank mPEG-DAS-APBA-Cou had lower toxicity. The formation of hemocompatible and cytocompatible polymers has been recognized as an essential characteristic to maintain their protein resistance properties ${ }^{57}$ and low cytotoxicity. Therefore, mPEG-DAS-APBA-Cou has better cytocompatibility for drug delivery.

The practicability of the mPEG-DAS-APBA-Cou micelles as a novel nanocarrier showed sensitivity to glucose and achieved controlled release of insulin. Insulin was loaded into mPEGDAS-APBA-Cou nanoparticles on the basis of hydrogen bonding, electrostatic and hydrophilic-hydrophobic interactions. The drug loading efficiency (DLE) and drug loading content (DLC) of the micelles are shown in Table 1 with respect to the standard curve of insulin. Fig. $4 \mathrm{D}$ exhibits the cumulative release of insulin-loaded polymers upon exposure to different glucose media $\left(0,1\right.$ and $\left.3 \mathrm{mg} \mathrm{mL}^{-1}\right)$ at $37{ }^{\circ} \mathrm{C}(\mathrm{pH}$ 7.4). The insulin was released from drug-loaded mPEG-DAS-APBA-Cou nanoparticles in the glucose-free medium because the nanoparticle surface was covered with insulin. With increasing glucose concentration ( 0 to $3 \mathrm{~g} \mathrm{~L}^{-1}$ ), the amount of released insulin significantly increased because of the higher glucose concentration. The more APBA moieties dissociated from the mPEG-DAS-APBA-Cou, the more the moieties were associated with higher concentrations of glucose. Compared with glucose in $1 \mathrm{mg} \mathrm{L^{-1 }}$ and $3 \mathrm{mg} \mathrm{L}^{-1}$ treatment, there was a close burst release phenomenon for MPEG-DAS-APBA-Cou within the first $5.0 \mathrm{~h}$, which was ascribed to the adsorption of insulin on the surface of the nanoparticles. Then, the insulin release rate became slow; it finally reached a plateau phase in the same time within $13.0 \mathrm{~h}$ and approximately increased by $25.0 \%$. At only $3 \mathrm{~g} \mathrm{~L}^{-1}$ glucose concentration, the structure of the nanoparticles was able to break and quickly release insulin. Compared with a report by Zhang et al..$^{58}$ on the cumulative release of insulinloaded polymers, mPEG-DAS-APBA-Cou showed a high amount of release in a short treatment time. 
A convenient technique, CD spectroscopy is the best way to evaluate the conformational changes and self-association of insulin. ${ }^{59}$ The ratio of the band at $208 \mathrm{~nm}$ arising from the $\alpha$-helix structure and that at $223 \mathrm{~nm}$ arising from the $\beta$-structure $\left([\phi]_{208} /[\phi]_{223}\right)$ can qualitatively measure the overall conformational structure of insulin (Fig. 6A). According to the spectral characteristics, the structure of the released insulin has not been distorted.

\section{Conclusions}

Novel amphiphilic glucose-sensitive dialdehyde starch polymer micelles with "shell-core" structures were synthesized by Schiff base bonds. Their DLC and DLE values were as high as 9.4\% and $30.4 \%$, respectively. Due to the carbohydrate moieties introduced into the polymers, the mPEG-DAS-APBA-Cou nanoparticles had low cytotoxic activity and hemolytic activity. The nanocarriers, whose $\mathrm{p} K_{\mathrm{a}}$ was reduced by the introduction of Cou, showed notable glucose responsive behaviour; they released insulin rapidly in $3 \mathrm{mg} \mathrm{mL} \mathrm{m}^{-1}$ glucose ( $\mathrm{pH}$ 7.4), with comparatively inert release in $1 \mathrm{mg} \mathrm{mL}^{-1}$ glucose ( $\mathrm{pH}$ 7.4). The glucose-sensitive insulin release could be adjusted by changing the glucose medium. Therefore, the amphiphilic mPEG-DASAPBA-Cou nanoparticles have potential applicability as a glucose-responsive material for insulin delivery.

\section{Conflicts of interest}

There are no conflicts to declare.

\section{Acknowledgements}

The authors gratefully acknowledge the financial support from the National Natural Science Foundation of China (grant no. 51541304, 51273086, 51503091, 51603097), the Special Doctorial Program Fund from the Ministry of Education of China (grant no. 20130211110017), and the Fundamental Research Funds for the Central Universities (grant no. lzujbky-2016-41, lzujbky-2017-it43).

\section{Notes and references}

1 K. M. Bratlie, R. L. York, M. A. Invernale, R. Langer and D. G. Anderson, Adv. Healthcare Mater., 2012, 1, 267-284.

2 I. G. Véronique Lapeyre, S. Chevreux and V. Ravaine, Biomacromolecules, 2006, 7, 3356-3363.

3 F. Cheng and F. Jäkle, Polym. Chem., 2011, 2, 2122.

4 W. Chen, Y. Cheng and B. Wang, Angew. Chem., 2012, 51, 5293-5295.

5 V. L. Alexeev, S. A. Asher, A. V. Goponenko, A. C. Sharma, I. K. Lednev, C. S. Wilcox and D. N. Finegold, J. Am. Chem. Soc., 2003, 125, 3322-3329.

6 Y. G. Yongjun Zhang and S. Zhou, Biomacromolecules, 2006, 7, 3196-3201.

7 R. Ma, H. Yang, Z. Li, G. Liu, X. Sun, X. Liu, Y. An and L. Shi, Biomacromolecules, 2012, 13, 3409-3417.
8 H. Yang, C. Zhang, C. Li, Y. Liu, Y. An, R. Ma and L. Shi, Biomacromolecules, 2015, 16, 1372-1381.

9 A. C. Sharma, V. L. Alexeev, A. V. Goponenko, S. Das, I. K. Lednev, C. S. Wilcox, D. N. Finegold and S. A. Asher, Anal. Chem., 2003, 75(10), 2316-2323.

10 Z. Wu, X. Zhang, H. Guo, C. Li and D. Yu, J. Mater. Chem., 2012, 22, 22788.

11 H. Yang, X. Sun, G. Liu, R. Ma, Z. Li, Y. An and L. Shi, Soft Matter, 2013, 9, 8589.

12 R. Ma, B. Wang, P. Sun and L. Shi, Chin. J. Chem., 2014, 32, 97-102.

13 J. Ren, Y. Zhang, J. Zhang, H. Gao, G. Liu, R. Ma, Y. An, D. Kong and L. Shi, Biomacromolecules, 2013, 14, 3434-3443.

14 H. Yang, R. Ma, J. Yue, C. Li, Y. Liu, Y. An and L. Shi, Polym. Chem., 2015, 6, 3837-3846.

15 R. Ma and L. Shi, Polym. Chem., 2014, 5, 1503-1518.

16 J. M. Harris and R. B. Chess, Nat. Rev. Drug Discovery, 2003, 2, 214-221.

17 C. Deng, Y. Jiang, R. Cheng, F. Meng and Z. Zhong, Nano Today, 2012, 7, 467-480.

18 J. N. Cambre, D. Roy, S. R. Gondi and B. S. Sumerlin, J. Am. Chem. Soc., 2007, 129(34), 10348-10349.

19 D. Roy and B. S. Sumerlin, ACS Macro Lett., 2012, 1, 529-532.

20 D. Roy, J. N. Cambre and B. S. Sumerlin, Chem. Commun., 2008, 2477-2479.

21 H. Kim, Y. J. Kang, E. S. Jeong, S. Kang and K. T. Kim, ACS Macro Lett., 2012, 1, 1194-1198.

22 H. Kim, Y. J. Kang, S. Kang and K. T. Kim, J. Am. Chem. Soc., 2012, 134, 4030-4033.

23 B. Wang, R. Ma, G. Liu, X. Liu, Y. Gao, J. Shen, Y. An and L. Shi, Macromol. Rapid Commun., 2010, 31, 1628-1634.

24 L. Zhao, C. Xiao, L. Wang, G. Gai and J. Ding, Chem. Commun., 2016, 52, 7633-7652.

25 A. Rodrigues and M. Emeje, Carbohydr. Polym., 2012, 87, 987-994.

26 G. H. Ahmed Besheer, J. Kressler and K. Mäder, Biomacromolecules, 2007, 8, 359-367.

27 H. Yamada, B. Loretz and C. M. Lehr, Biomacromolecules, 2014, 15, 1753-1761.

28 M. J. Santander-Ortega, T. Stauner, B. Loretz, J. L. OrtegaVinuesa, D. Bastos-Gonzalez, G. Wenz, U. F. Schaefer and C. M. Lehr, J. Controlled Release, 2010, 141, 85-92.

29 H. Horchani, M. Chaâbouni, Y. Gargouri and A. Sayari, Carbohydr. Polym., 2010, 79, 466-474.

30 L. Zhang, X. Gong, Y. Wang and H. Qu, J. Chem. Eng. Data, 2012, 57, 2018-2022.

31 S. H. Yoo, J. S. Lee, S. Y. Park, Y. S. Kim, P. S. Chang and H. G. Lee, Int. J. Biol. Macromol., 2005, 35, 27-31.

32 Q. Wang, C. X. Li, X. Fan, P. Wang and L. Cui, Biocatal. Biotransform., 2009, 26, 437-443.

33 X. He, M. Du, H. Li and T. Zhou, Int. J. Biol. Macromol., 2016, 82, 174-181.

34 J.-Y. Kim and S.-T. Lim, Carbohydr. Polym., 2009, 76, 110116.

35 V. Mazíková, I. Sroková and A. Ebringerová, Chem. Pap., 2009, 63(1), 71-76. 
36 Z. Zhang, H. Shan, L. Chen, C. He, X. Zhuang and X. Chen, Eur. Polym. J., 2013, 49, 2082-2091.

37 Y. M. Daijiro Shiino, A. Kubo, Y. J. Kim, K. Kataoka, Y. Koyama, A. Kikuchi, M. Yokoyama, Y. Sakurai and T. Okano, J. Controlled Release, 1995, 37, 269-276.

38 S. I. Akira Matsumoto, A. Harada and K. Kataoka, Biomacromolecules, 2003, 4, 1410-1416.

39 J. Yoon and A. W. Czarnik, J. Am. Chem. Soc., 1992, 114, 58745875.

40 N. M. Weitai Wu, E. C. Y. Yan and S. Zhou, ACS Nano, 2010, 4, 4831-4839.

41 Z. Yang, W. Zhang, J. Zou and W. Shi, Polymer, 2007, 48, 931938.

42 A. Zhang, Z. Zhang, F. Shi, J. Ding, C. Xiao, X. Zhuang, C. He, L. Chen and X. Chen, Soft Matter, 2013, 9, 2224.

43 N. L. Chun Zhang and D. E. Hirt, Langmuir, 2006, 22, 68516857.

44 M. Chen, C. Gao, S. Lü, Y. Chen and M. Liu, RSC Adv., 2016, 6, 46159-46169.

45 M. Badawi, S. Soundararajan, C. Montafio Kohlrust and J. H. Hagernan, Anal. Biochem., 1989, 178, 125-134.

46 H. Liu, D. Chaudhary, S.-i. Yusa and M. O. Tadé, Carbohydr. Polym., 2011, 83, 1591-1597.

47 J. Yang, C. Gao, S. Lü, X. Wang, M. Chen and M. Liu, RSC Adv., 2014, 4, 55139-55149.
48 M. G. Mohamed, K.-C. Hsu and S.-W. Kuo, Polym. Chem., 2015, 6, 2423-2433.

49 W. Yu, H. Zhou, X. Guo, X. Liu, N. Li, Y. Zhang and X. Ma, Biomacromolecules, 2010, 11, 3480-3486.

50 H. Zhang, X. Kong, Y. Tang and W. Lin, ACS Appl. Mater. Interfaces, 2016, 8, 16227-16239.

51 H. Deng, X. Zhao, J. Liu, J. Zhang, L. Deng, J. Liu and A. Dong, Nanoscale, 2016, 8, 1437-1450.

52 G. S. a. B. Wang, Tetrahedron, 2002, 58, 5291-5300.

53 A. Yildirim, E. Ozgur and M. Bayindir, J. Mater. Chem. B, 2013, 1, 1909.

54 M. S. W. Mingchao Shen, D. G. Castner, B. D. Ratner and T. A. Horbett, Langmuir, 2003, 19, 1692-1699.

55 D. Kwak, Y. Wu and T. A. Horbett, J. Biomed. Mater. Res., Part A, 2005, 74, 69-83.

56 D. Eglin, S. Grad, S. Gogolewski and M. Alini, J. Biomed. Mater. Res., Part A, 2010, 92, 393-408.

57 Y. J. Shih, Y. Chang, A. Deratani and D. Quemener, Biomacromolecules, 2012, 13, 2849-2858.

58 G. Zhang, X. Zhang, H. Shen, J. Yang and J. Yang, RSC Adv., 2014, 4, 49964-49973.

59 K. Kim, S. Lee, T. Suresh Kumar, J. Lee, S. Kyoon Kim, D. Yun Lee, Y.-k. Lee and Y. Byun, Bioconjugate Chem., 2005, 16, 615620. 